\title{
Análise espacial da tuberculose em Belém, estado do Pará, Brasil
}

\author{
Spatial analysis of tuberculosis in Belém, Pará State, Brazil
}

Sandra Souza Lima, Antonio Carlos Rosário Vallinoto, Luiz Fernando Almeida Machado, Marluísa de Oliveira Guimarães Ishak, Ricardo Ishak

Universidade Federal do Pará, Instituto de Ciências Biológicas, Laboratório de Virologia, Belém, Pará, Brasil

\begin{abstract}
RESUMO
INTRODUÇÃO: A tuberculose (TB), no Brasil, concentra-se nas regiões metropolitanas e é associada à situação socioeconômica da população. Em 2011, Belém, capital do estado do Pará, apresentou uma das maiores baixas de incidência da doença. OBJETIVOS: Avaliar a distribuição espacial da infecção pelo Mycobacterium tuberculosis em Belém entre 2006 e 2010, e associar a incidência da infecção com as condições de vida da população. MATERIAIS E MÉTODOS: Informações de morbidade e mortalidade foram obtidas de bancos de dados nacionais (SINAN, SIM e IBGE). Os índices de Moran global (IMG) e Moran local identificaram dependência espacial. RESULTADOS: A taxa de incidência da TB alcançou 93 casos/100.000 habitantes e a de mortalidade $4 \mathrm{casos} / 100.000$ habitantes. O IMG mostrou dependência espacial negativa quanto à incidência e à dependência espacial positiva nas taxas de mortalidade entre bairros. A incidência de casos de TB cresceu com o aumento do número de áreas carentes nos bairros. O método bayesiano foi eficiente para analisar a incidência da doença em bairros com populações pequenas. A incidência se distribuiu espacialmente de forma aleatória e associada às condições socioeconômicas da população. A baixa taxa de mortalidade evidenciou uma boa avaliação dos serviços de tratamento e acompanhamento dos doentes. CONCLUSÃO: A aplicação da análise espacial e métodos estatísticos, que aprimoram a qualidade da informação, são importantes para melhor avaliar ações futuras de prevenção contra agentes infecciosos. É necessário dar continuidade a campanhas de prevenção da TB e ao acompanhamento de doentes, a fim de aumentar a adesão ao tratamento e diminuir a mortalidade entre a população com maior dificuldade de acesso aos serviços de saúde.
\end{abstract}

Palavras-chave: Tuberculose; Análise Espacial; Método Bayesiano.

\begin{abstract}
INTRODUCTION: Tuberculosis (TB) in Brazil is mainly located in major urban centers and is associated with the social and economical patterns. In 2011, Belém, the capital of Pará State, showed one of the highest incidence. OBJECTIVES: To investigate the spatial distribution of Mycobacterium tuberculosis in Belém between 2006 and 2010, and to associate the incidence of infection with the life quality of the population. MATERIALS AND METHODS: Morbidity and mortality information were obtained from national public information sources (SINAN, SIM and IBGE). Global Moran's index (GMI) and local Moran's index were used to identify spatial associations. RESULTS: Incidence rate of TB was 93 cases/100,000 inhabitants and mortality reached 4 cases/100,000. GMI showed negative space dependence with regard to incidence and positive space dependence in mortality rates among districts. Incidence of TB showed an increase according to the poorest quality of life areas of the city. The Bayesian method was successful to analyze the incidence of TB in low population density areas. The incidence was spatially distributed randomly and associated with the socioeconomic conditions of population. The low mortality rate was an evidence of the good treatment services and follow up of the patients. CONCLUSION: The use of spatial analysis and statistical methods, that improve the quality of the information, are important to better evaluate the future prevention actions against infectious agents. It is necessary to continue with TB prevention campaigns and patient follow-up in order to increase adherence to treatment and decrease mortality among the population with the greatest difficulty in accessing health services.
\end{abstract}

Keywords: Tuberculosis; Spatial Analysis; Bayesian Method.

\footnotetext{
Correspondência / Correspondence:

Ricardo Ishak

Universidade Federal do Pará, Instituto de Ciências Biológicas, Laboratório de Virologia

Rua Augusto Corrêa, s/n. Bairro: Guamá - CEP: 66075-910 - Belém, Pará, Brasil - Tel.: +55 (91) 3201 -7587

E-mail: rishak@ufpa.br
} 


\section{INTRODUÇÃO}

A tuberculose (TB), doença infecciosa causada pelo Mycobacterium tuberculosis, ainda é considerada um grave problema de saúde pública mundial, apesar de apresentar uma tendência decrescente nos últimos anos no mundo'.

Ainda que os números mostrem uma redução no número de casos, o Brasil ocupa o $20^{\circ}$ lugar entre os países com maior número de casos de TB no mundo, com uma taxa de incidência de 46 casos por 100.000 habitantes $^{1}$ e maior concentração nas capitais e regiões metropolitanas ${ }^{2,3}$. Em 2011, uma das capitais com maior incidência foi Belém, no estado do Pará, que apresentou uma taxa de 84,9 casos por 100.000 habitantes ${ }^{4}$.

A análise da distribuição espacial de casos de TB, dentro de um mesmo município, é fundamental para a identificação de áreas de risco e de bolsões de subnotificações intramunicipais ${ }^{5,6}$. A heterogeneidade na distribuição espacial da TB é frequentemente descrita de acordo com os municípios, para ressaltar essa característica $7,8,9,10,11$.

A ocorrência de TB, tanto individual quanto de forma coletiva, é consistentemente associada a indicadores socioeconômicos ${ }^{12}$. Essa associação parece ser influenciada tanto pelo nível de agregação espacial quanto pelas características particulares das áreas geográficas.

presente estudo teve como objetivos descrever - padrão espacial da infecção pelo M. tuberculosis, em Belém, e identificar a associação da incidência da infecção com as condições da vida social e econômica da população.

\section{MATERIAIS E MÉTODOS}

Foi realizado um estudo descritivo ecológico para avaliar a distribuição espacial da TB (incidência e mortalidade) e associá-la com as condições sociodemográficas dos moradores, por bairro de residência, em Belém, no período de 2006 a 2010.

\section{ÁREA DE ESTUDO E POPULAÇÃO}

○ município de Belém, por ocasião do estudo, possuía uma população de 1.393.399 habitantes $^{13}$, distribuída em uma área de $1.059 .402 \mathrm{~km}^{2}$, o que representava uma densidade demográfica de 1,3 habitantes $/ \mathrm{km}^{2}$. A cidade é dividida em oito distritos administrativos e 71 bairros. $\bigcirc$ número médio de moradores era de 3,8 por domicílio e o índice de desenvolvimento humano de 0,746, considerado alto.

Os dados de morbidade foram obtidos do Sistema de Informação de Agravos de Notificação (SINAN) e os dados de mortalidade, tendo como causa básica a TB, do Sistema de Informação sobre Mortalidade (SIM) do Ministério da Saúde. As informações sociais, demográficas, econômicas e a malha geográfica dos bairros de Belém foram obtidas do Instituto Brasileiro de Geografia e Estatística (IBGE). critério de definição de caso novo incluiu pacientes que nunca se submeteram à quimioterapia antituberculose ou que fizeram terapia por menos de 30 dias ou há mais de cinco anos. Todos os pacientes tinham residência fixa em Belém.

Não fizeram parte do estudo indivíduos moradores de rua e institucionalizados (residentes em presídios, asilos e casas de apoio), por possuírem um risco de infecção e de doença maior que a população em geral. Foram também excluídos indivíduos que não tinham endereço completo, o que não permitia identificar o bairro de residência.

\section{ANÁLISE ESTATÍSTICA E ESPACIAL}

Foi aplicada a técnica de análise espacial de dados agregados por áreas, utilizando-se, como unidade de análise, os bairros de residência dos indivíduos. A partir das bases de dados do SINAN e do SIM, foram calculadas as taxas de incidência e de mortalidade da TB de acordo com o bairro. A taxa de incidência foi calculada ano a ano, enquanto que a taxa de mortalidade foi calculada para o período total estudado. Para a análise de mortalidade, alguns bairros foram agregados a outros bairros vizinhos e pertencentes ao mesmo distrito administrativo. A agregação se fez necessária para diminuir a instabilidade do indicador, devido à ocorrência de óbitos ser baixa.

Em seguida, as taxas foram suavizadas pelo estimador bayesiano empírico, para reduzir a variabilidade aleatória e proporcionar uma maior estabilidade dos indicadores $^{14}$.

Para verificar a autocorrelação espacial entre as taxas suavizadas, foi utilizado o índice de Moran global (IMG). A identificação de áreas críticas ou de transição foi avaliada pelo índice de Moran local (IML), para comparar o valor de cada bairro estudado com bairros vizinhos e exibir dependência e padrões espaciais ${ }^{15}$. Os quadrantes gerados foram analisados da seguinte forma:

- Q1 (valores positivos, médias positivas) e Q2 (valores negativos, médias negativas) - indicaram pontos de associação espacial positiva, no sentido que uma localização possui vizinhos com valores semelhantes;

-Q3 (valores positivos, médias negativas) e Q4 (valores negativos, médias positivas) - indicaram pontos de associação espacial negativa, no sentido que uma localização possui vizinhos com valores distintos.

Para a representação espacial das áreas críticas, foi utilizado o Moran Map, considerando-se apenas os valores estatisticamente significativos $(p \leq 0,05)$. Os bairros críticos, com risco elevado de incidência/ mortalidade por TB, foram os classificados no quadrante Q1 do Moran Map.

$O$ teste de McNemar foi aplicado para identificar se houve mudança na classificação dos bairros, em relação ao nível de prioridade, do ano de 2006 para o ano de 2010. 


\section{ANÁLISE SOCIOECONÔMICA}

A identificação de áreas carentes no município seguiu o critério do IBGE, o qual considera a presença de características, como: existência de unidades habitacionais que ocupam ou tenham ocupado terreno de propriedade alheia há pelo menos 10 anos; urbanização desordenada e densa; e precariedade de serviços públicos essenciais, como saneamento, energia elétrica e abastecimento de água ${ }^{16}$. Foi relacionado o número de áreas carentes em cada bairro com a incidência e a mortalidade por TB, para avaliar a relação desses indicadores com as condições de vida da população.

A análise estatística espacial foi realizada utilizando-se os softwares BioEstat v5.0'17 e TerraView v4.2.2 do Instituto Nacional de Pesquisa Espacial do Brasil. Os mapas foram gerados por meio do programa ArcMap 10.

\section{ASPECTOS ÉTICOS}

estudo foi aprovado pela Comissão de Ética em Pesquisa do Hospital Universitário João de Barros Barreto da Universidade Federal do Pará, em 2 de outubro de 2010 (processo $n^{\circ} 2825 / 10$ ).

\section{RESULTADOS}

\section{INCIDÊNCIA DE CASOS DE TB}

Durante o período estudado, foram notificados 7.444 casos de TB em indivíduos residentes no município de Belém, dos quais 6.541 (87,9\%) foram de casos novos, representando uma incidência de 93 casos por 100.000 habitantes. As taxas de incidência nos anos de 2006 e 2010 foram, respectivamente, 84,8 e 98,4 por 100.000 habitantes, representando um aumento de $16 \%$.

As médias da taxa bruta e da taxa suavizada da incidência de casos de TB mostraram tendência de crescimento gradativo ao longo dos anos avaliados e a existência de grande variação das taxas entre os bairros
(Tabela 1). Nas duas taxas, o menor valor de incidência ocorreu no ano de 2006 e o maior, no ano de 2009. A taxa suavizada, em todos os anos, foi maior que a taxa bruta, porém a variação da taxa suavizada foi menor $(23,0$ vs. 15,7).

Os bairros com as maiores taxas de incidência suavizada foram Águas Lindas (275,4\%), Una (245,2\%), Coqueiro (243,7\%), Maracangalha $(159,2 \%)$, São Francisco (148,4\%), Parque Guajará (144,4\%) e Praia Grande (132,8\%). Os bairros que apresentaram as menores taxas de incidência suavizada foram Agulha $(-67,8 \%)$, Souza $(-48,8 \%)$, Maracacuera $(-26,1 \%)$, Canudos (-25,4\%), Fátima $(-25,0 \%)$ e Baia do Sol $(-21,3 \%)$.

IMG apresentou valores de $-0,12(p=0,12)$ em 2006, $-0,15(p=0,01)$ em 2007, $-0,13 \quad(p=0,03)$ em 2008, -0,10 (p=0,02) em 2009 e $-0,15$ $(p=0,05)$ em 2010, que evidenciaram dependência espacial negativa significativa para os anos de 2007 a 2010 (mas não para 2006), demonstrando que os bairros com altas taxas de incidência estão cercados por bairros de baixas taxas, confirmando a distribuição espacial aleatória da doença nos bairros.

A tabela 2 mostra a frequência dos pontos de associação espacial, positiva e negativa, evidenciando que, ao longo dos anos, houve um aumento no número de bairros classificados no quadrante Q2 e uma diminuição do número de bairros no quadrante Q1. Ainda assim, a taxa de incidência de casos de TB mostrou um aumento em todos os quadrantes, em relação ao ano de 2006. As frequências das taxas de incidência dos quadrantes Q1 e Q3 foram as mais altas e as dos quadrantes $Q 2$ e Q4, as mais baixas.

Nos quadrantes Q1 e Q3, estão os bairros com as maiores taxas de incidência do Município, que são os bairros de Val-de-Cans $(304,0)$, Jurunas $(148,0)$, Cremação (144,0), Fátima (144,0), entre outros.

Tabela 1 - Estatística descritiva das taxas de incidências bruta e suavizada da TB, por 100.000 habitantes, em Belém, estado do Pará, Brasil, no período de 2006 a 2010

\begin{tabular}{|c|c|c|c|c|c|c|c|c|}
\hline \multirow[b]{2}{*}{ Ano } & \multicolumn{4}{|c|}{ Taxa bruta } & \multicolumn{4}{|c|}{ Taxa suavizada } \\
\hline & Média & $\begin{array}{l}\text { Desvio- } \\
\text { padrão }\end{array}$ & Mínimo & Máximo & Média & $\begin{array}{l}\text { Desvio- } \\
\text { padrão }\end{array}$ & Mínimo & Máximo \\
\hline 2006 & 72,26 & 68,80 & - & 319,45 & 81,08 & 44,03 & 8,33 & 246,06 \\
\hline 2007 & 86,23 & 76,14 & - & 345,11 & 86,84 & 42,34 & 15,73 & 254,20 \\
\hline 2008 & 88,40 & 88,00 & - & 467,30 & 91,49 & 47,50 & 12,34 & 313,23 \\
\hline 2009 & 97,00 & 135,30 & - & $1.028,80$ & 97,53 & 61,26 & 11,12 & 415,52 \\
\hline 2010 & 95,30 & 91,60 & - & 534,80 & 96,09 & 40,95 & 21,58 & 304,08 \\
\hline
\end{tabular}


Tabela 2 - Frequência do número de bairros e da taxa de incidência de TB, por 100.000 habitantes, em Belém, estado do Pará, Brasil, no período de 2006 a 2010, de acordo com o quadrante definido IML

\begin{tabular}{|c|c|c|c|c|c|c|}
\hline \multirow{2}{*}{ Ano } & \multirow{2}{*}{ Indicadores } & \multicolumn{5}{|c|}{ Quadrantes } \\
\hline & & Q1 (+/+) & Q2 (-/-) & Q3 (+/-) & Q4 (-/+) & Total \\
\hline \multirow{3}{*}{2006} & $N^{\circ}$ de bairros & 11 & 17 & 19 & 24 & 71 \\
\hline & $\%$ & 15,5 & 23,9 & 26,8 & 33,8 & 100,0 \\
\hline & Taxa de incidência & 112,46 & 54,38 & 123,62 & 51,94 & \\
\hline \multirow{3}{*}{2007} & $\mathrm{~N}^{\circ}$ de bairros & 9 & 18 & 21 & 23 & 71 \\
\hline & $\%$ & 12,7 & 25,3 & 29,6 & 32,4 & 100,0 \\
\hline & Taxa de incidência & 130,60 & 64,76 & 120,26 & 56,47 & \\
\hline \multirow{3}{*}{2008} & $\mathrm{~N}^{\circ}$ de bairros & 10 & 22 & 16 & 23 & 71 \\
\hline & $\%$ & 14,1 & 31,0 & 22,5 & 32,4 & 100,0 \\
\hline & Taxa de incidência & 131,83 & 69,98 & 141,05 & 60,06 & \\
\hline \multirow{3}{*}{2009} & $\mathrm{~N}^{\circ}$ de bairros & 7 & 25 & 17 & 22 & 71 \\
\hline & $\%$ & 9,9 & 35,2 & 23,9 & 31,0 & 100,0 \\
\hline & Taxa de incidência & 145,50 & 72,63 & 158,62 & 63,33 & \\
\hline \multirow{3}{*}{2010} & $\mathrm{~N}^{\circ}$ de bairros & 8 & 21 & 19 & 23 & 71 \\
\hline & $\%$ & 11,2 & 29,6 & 26,8 & 32,4 & 100,0 \\
\hline & Taxa de incidência & 133,59 & 75,59 & 133,43 & 70,90 & \\
\hline
\end{tabular}

Apenas um bairro, por ano, apresentou uma taxa de incidência que poderia ser considerada como de maior gravidade epidemiológica, de acordo com a situação no quadrante Q1 e pelo Moran Map (Figura 1): em 2006, foi o bairro da Pedreira; em 2007, 2009 e 2010, o da Sacramenta. Em 2008, não foi identificada significância em qualquer bairro.

A evolução da classificação dos bairros, de acordo com o quadrante, mostrou que, dentre os 71 bairros de Belém, 21 (29,6\%) permaneceram com taxas de incidência altas, $35(49,3 \%)$ permaneceram com taxas baixas, nove $(12,7 \%)$ tiveram diminuição das taxas e seis $(8,4 \%)$ tiveram aumento das taxas. Não ocorreram mudanças nas taxas de incidência que fossem estatisticamente significativas no período estudado.

\section{MORTALIDADE POR TB}

Durante o período analisado, foram notificados 258 óbitos por TB entre a população residente em Belém, o que corresponde a uma taxa de mortalidade bruta de quatro casos por 100.000 habitantes. Com a aplicação da suavização da taxa de mortalidade, observou-se uma diminuição na variação das mesmas. $\bigcirc$ valor máximo da taxa de mortalidade bruta foi de 16,1 por 100.000 habitantes e, na taxa suavizada, foi de 5,2 por 100.000 habitantes.

Após a suavização da taxa de mortalidade, os cinco bairros com maiores índices foram Pedreira $(5,2)$, Pratinha $(5,1)$, Sacramenta $(5,0)$, Val-de-Cans $(4,9)$ e Telégrafo $(4,9)$.
O IMG apresentou valor de 0,52 $(p=0,01)$, e a distribuição do número de bairros, por quadrante, mostrou Q1 (42; 59,1\%), Q2 (19; 26,8\%), Q3 $(0 ; 0,0 \%)$ e $Q 4(10 ; 14,1 \%)$. A maioria dos bairros estava concentrada nos quadrantes Q1 e Q2, o que demonstrou a existência de dependência espacial positiva das taxas de mortalidade. Em relação à taxa de mortalidade por 100.000 habitantes, por quadrante, obteve-se Q1 (4,3), Q2 $(2,6)$, Q3 $(0,0)$ e Q4 $(3,2)$.

A figura 2 mostra uma área crítica (Q1) formada por cinco bairros (Fátima, Pedreira, Sacramenta, Umarizal e Telégrafo) e uma grande área (Q2) formada por bairros de taxa de mortalidade baixa.

Quanto às condições de vida da população, foram identificadas 71 áreas carentes, com 54,9\% residente nessas áreas. Conforme demonstrado na tabela 3, o maior número de bairros $(44 ; 62,0 \%)$ apresentou poucos pontos de áreas carentes (zero a cinco) e menor concentração populacional (23,5\%). O maior número de notificações e a maior taxa de incidência de casos de TB aumentaram gradativamente com o aumento do número de áreas carentes e de concentração populacional. A maior taxa de incidência (1 19,65/100.000 habitantes) ocorreu entre - maior agrupamento populacional e onde mais se encontravam pontos de áreas carentes, ainda que em um número menor de bairros (16,9\%). Aparentemente, quando o número de áreas carentes aumenta, a taxa de incidência também aumenta. A taxa de mortalidade não segue esse padrão. 
2006
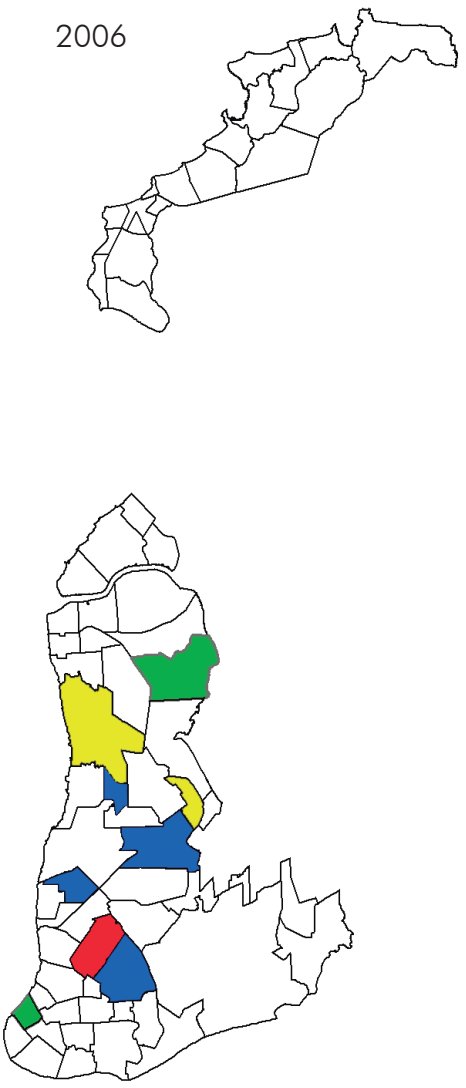

2009

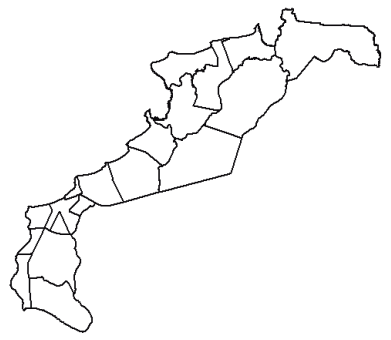

2007
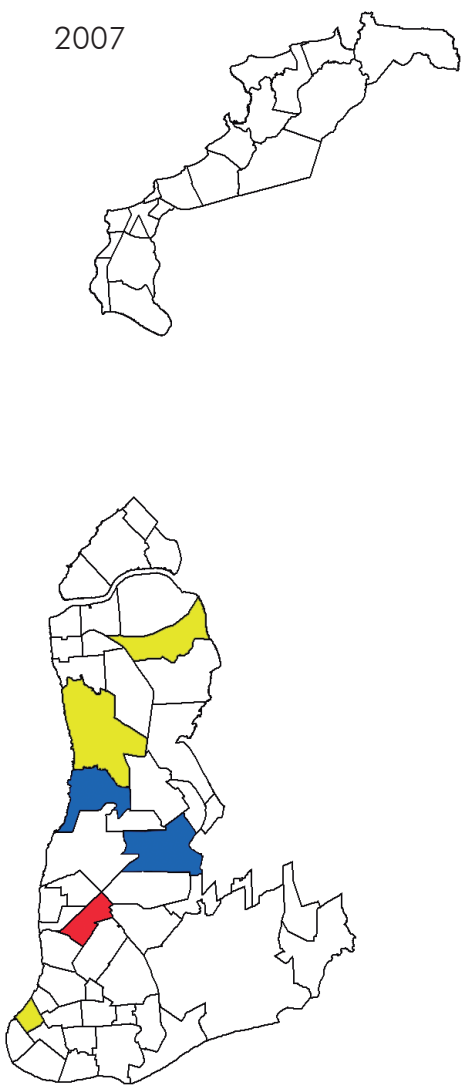

2010

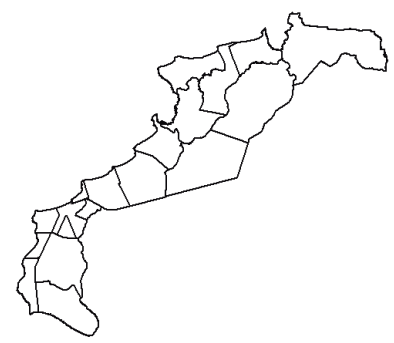

2008
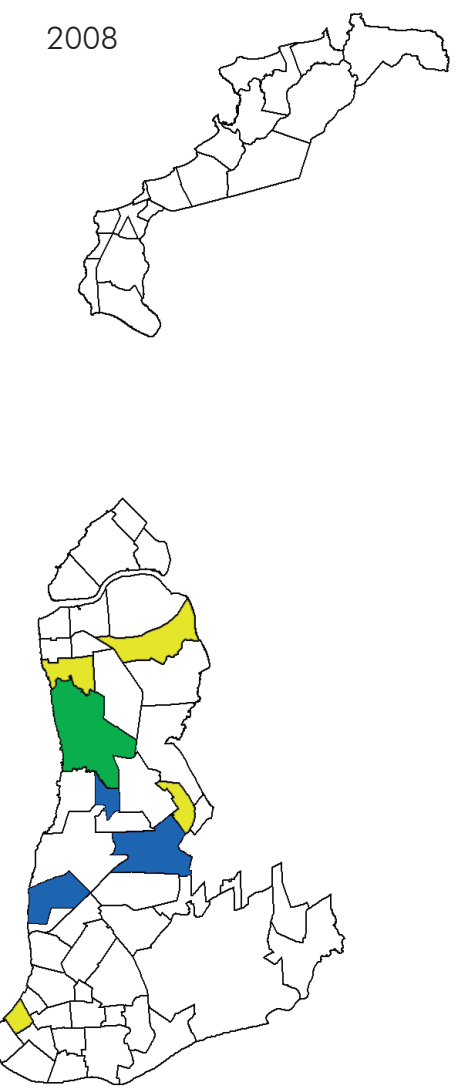

Não significante

Q1 (+/+)

Q2 (-/-)

Q3 (+/-)

Q4 (-/+)

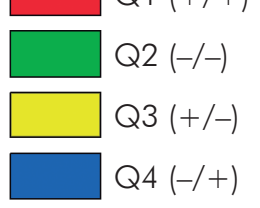

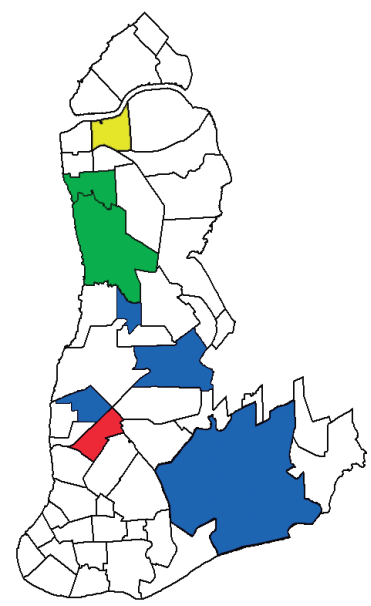

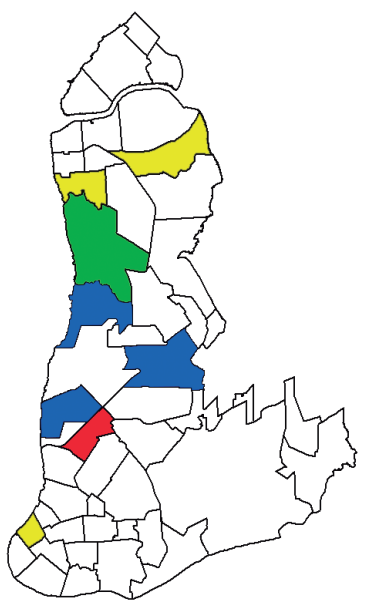

Figura 1 - Representação da distribuição espacial da taxa de incidência suavizada de casos de TB em Belém, estado do Pará, Brasil, de 2006 a 2010, usando o Moran Map 

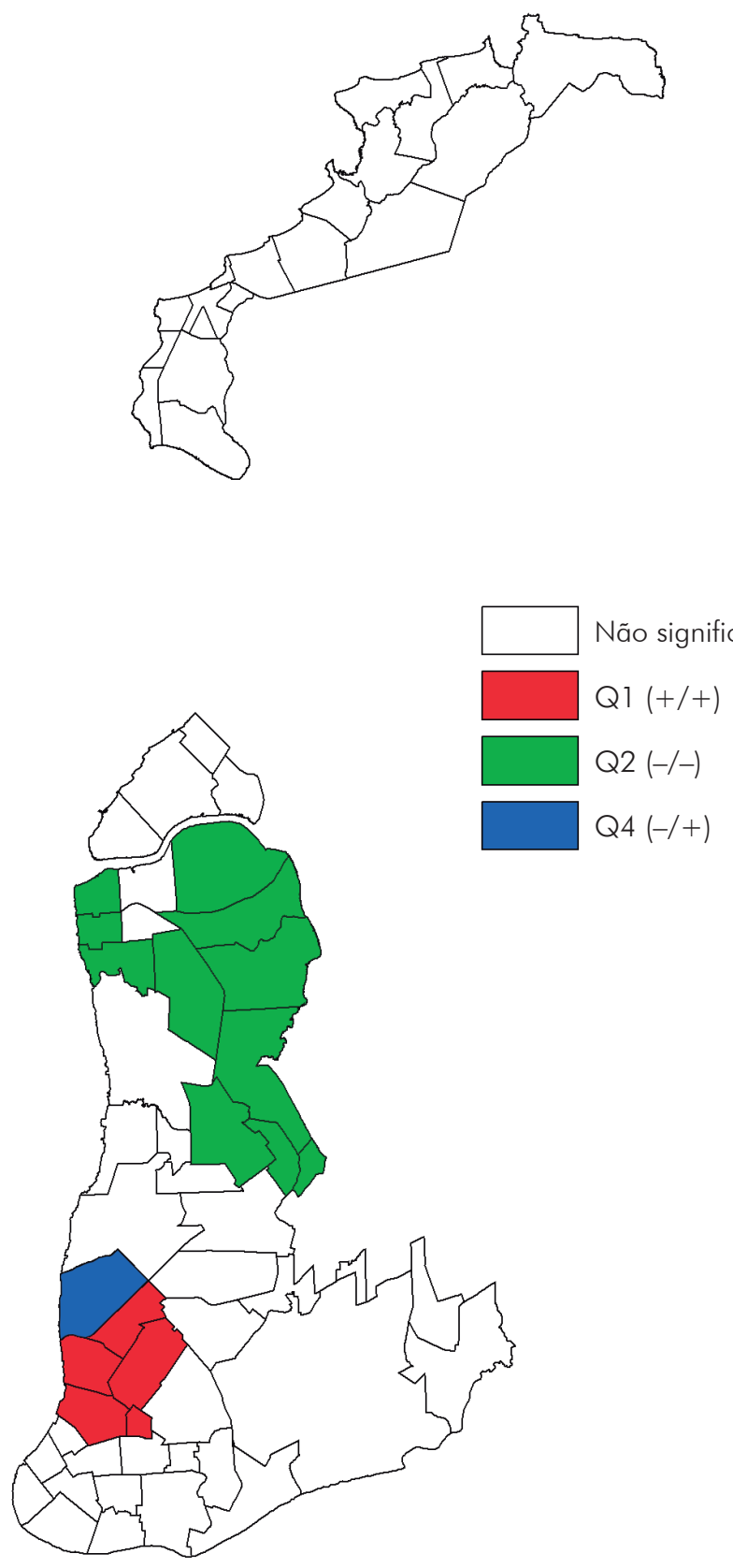

Figura 2 - Representação da distribuição espacial da taxa de mortalidade suavizada de casos de TB em Belém, estado do Pará, Brasil, de 2006 a 2010, usando o Moran Map

Tabela 3 - Comparação entre os indicadores de incidência e de mortalidade por TB, por 100.000 habitantes, com o número de áreas carentes nos bairros de Belém, estado do Pará, Brasil, em 2010

\begin{tabular}{cccccc}
\hline $\begin{array}{c}N^{\circ} \text { de áreas } \\
\text { carentes }\end{array}$ & $\begin{array}{c}\text { Número de bairros } \\
(\%)\end{array}$ & População & $\begin{array}{c}\text { No de notificações } \\
\text { de TB }\end{array}$ & $\begin{array}{c}\text { Taxa de incidência } \\
\text { de TB }\end{array}$ & $\begin{array}{c}\text { Taxa de } \\
\text { mortalidade }\end{array}$ \\
\hline 0 & $22(31,0)$ & 139.872 & 96 & 68,63 & 3,57 \\
1 a 5 & $22(31,0)$ & 184.565 & 161 & 87,23 & 2,71 \\
6 a 20 & $15(21,1)$ & 453.623 & 380 & 83,77 & 3,74 \\
21 a 84 & $12(16,9)$ & 603.415 & 722 & 119,65 & 2,82 \\
\hline Total & $71(100,0)$ & 1.381 .475 & 1.359 & 98,37 & 3,18 \\
\hline
\end{tabular}




\section{DISCUSSÃO}

Apesar da incidência da TB, no Brasil, apresentar um declínio nos últimos anos, Belém apresentou uma tendência crescente e uma incidência média duas vezes superior à incidência observada no estado do Pará, quase três vezes maior que a média no Brasil ${ }^{4}$, evidenciando a concentração de casos nessa capital ${ }^{4,18}$, - que também ocorre em outros estados brasileiros 6 . Essas características podem ser uma consequência da alta densidade demográfica e dos bolsões de pobreza característicos dos grandes centros urbanos, além de ser, nas capitais do país, onde existem maior oferta dos serviços de saúde e melhores condições de diagnóstico e tratamento para os casos da doença ${ }^{3}$, aumentando o número de registros de notificações.

$\mathrm{Na}$ variação da incidência suavizada da TB, por bairro, observou-se que, em Águas Lindas, Una e Coqueiro, ocorreu uma variação superior à variação da incidência do Município. Esses bairros não apresentaram uma alta incidência, mas mostraram um crescimento expressivo ao longo dos anos. Entretanto, outros bairros, como Agulha, Souza e Maracacuera, apresentaram diminuição da taxa de incidência. É possível que tenha ocorrido alguma ação de saúde que justifique a diminuição de casos, podendo ter havido também subnotificação da doença.

A partir dos resultados da análise espacial, não foi identificado agrupamento de bairros com taxas de incidências similares, o que indica que casos da doença não estão concentrados em uma única região, mas dispersos por todo o Município. Essa heterogeneidade da distribuição espacial da TB reforça a necessidade de implementar ações diferenciadas, dirigidas a grupos específicos, para a prevenção e controle da TB dentro de um mesmo município. A heterogeneidade na distribuição espacial da TB foi também identificada em outros municípios brasileiros $7,19,20,21$.

Vários estudos mostraram, pelo IMG, que a distribuição espacial da TB possui autocorrelação positiva, indicando que áreas com taxas de incidência semelhantes tendem a estar agrupadas $21,22,23,24,25$. Em Belém, essa situação não foi encontrada, sendo que os bairros que apresentaram taxas de incidência semelhantes não estavam concentrados, mas espalhados por toda a cidade, mostrando uma autocorrelação negativa. Essa forma de distribuição espacial de propagação da doença mostra-se diferenciada e pode estar relacionada com a similaridade das condições de vida nos bairros de Belém.

A grande variação da taxa de incidência entre os bairros, mesmo depois da suavização, indica a diferença na situação de risco entre eles. Alguns bairros apresentaram incidências similares ou menores que a incidência nacional, mas outros, como Val-de-Cans, Jurunas e Cremação, evidenciaram incidência próxima a de países com as maiores taxas de incidência do mundo, como Filipinas, Etiópia, Moçambique e Bangladesh?.
Quando foi avaliada a classificação de prioridade pelo IML nos anos estudados, verificou-se que alguns bairros mudaram a condição de prioridade; alguns saíram da condição de maior prioridade e outros passaram para a condição de prioritários. Apesar de não haver um padrão de autocorrelação espacial global, o IML identificou áreas prioritárias no combate à TB e, a cada ano, um bairro foi identificado como prioritário. Essa é uma evidência clara das disparidades intraurbanas na distribuição da TB.

As medidas de avaliação e monitoramento utilizadas no presente trabalho, como: (i) a identificação de bairros com maior risco de incidência de casos de TB que utiliza a taxa de incidência suavizada; (ii) o monitoramento continuo da evolução da incidência suavizada, por bairro, ano a ano, para analisar a tendência de crescimento; e (iii) a análise da distribuição espacial para verificar a correlação entre os bairros vizinhos, são importantes dispositivos no controle da TB, pois permitem a interferência dos gestores de saúde na prevenção e controle da doença e uma ação de controle pontual e imediata nesses bairros.

diagnóstico tardio é um dos principais fatores que contribuem para o agravamento da TB e, consequentemente, $O$ óbito ${ }^{26,27}$. A alta taxa de mortalidade por TB, em um bairro, indica a falta de informação e de capacidade do sistema de vigilância e controle em identificar os casos antes do agravamento da doença.

Em Belém, a taxa de mortalidade por TB foi superior às taxas estadual e nacional ${ }^{4,18}$, demonstrando que a ocorrência do óbito por TB está mais concentrada em Belém do que nos outros municípios do estado do Pará.

A distribuição dos bairros entre os quadrantes evidencia uma grande variabilidade em relação à taxa de mortalidade. A taxa de mortalidade do quadrante de maior taxa é 13 vezes maior que o quadrante com menor taxa. Essa diferença, dentro de um mesmo município, foi encontrada por outros autores ${ }^{28}$ e pode estar relacionada à dificuldade de acesso aos serviços de saúde e à falta de adesão ao tratamento de pacientes em alguns bairros, demonstrando a necessidade de ações imediatas de educação e vigilância.

A TB é um dos agravos fortemente influenciados pelas variáveis sociais e demonstra relação direta com indicadores de pobreza e exclusão social no Brasil ${ }^{18}$. Outros autores já utilizaram diversas técnicas de análise espacial para identificar a associação da TB com fatores socioeconômicos e concluíram a existência de dependência da TB com a situação social da população $7,9,10,11,22$.

Uma das características dominantes em Belém é a grande extensão de áreas carentes que não dispõem de serviços essenciais adequados, distribuídas de forma desordenada pelo Município. As mais antigas localizam-se próximas ao centro comercial, o qual está sujeito a inundações periódicas, e caracterizam-se por 
elevada densidade demográfica. Os bairros mais novos estão localizados ao norte do Município, onde existem grandes ocupações, as quais estão próximas a áreas similares no município de Ananindeua ${ }^{16}$.

Belém apresenta também, como característica peculiar, possuir bairros com grande heterogeneidade social, o que fica evidente na distribuição espacial da TB. Essa heterogeneidade social foi evidenciada quando o número de áreas carentes foi associado às taxas de incidência e mortalidade por TB. Verificou-se que, quanto maior o número de áreas carentes nos bairros, maior foi a incidência de casos de TB. Tal situação já havia sido demonstrada em locais como São José do Rio Preto ${ }^{11,22}$ e Ribeirão Preto, 9 , no estado de São Paulo.

\section{CONCLUSÃO}

A aplicação da análise espacial, associada a métodos estatísticos que melhoram a qualidade da informação, é uma ferramenta que deve ser aplicada para melhor avaliar as ações futuras de prevenção contra qualquer agente infeccioso. A continuidade de campanhas de prevenção da doença e de acompanhamento é necessária para aumentar a adesão ao tratamento e diminuir a mortalidade entre a população com maior dificuldade de acesso aos serviços de saúde.

método bayesiano, aplicado nas taxas de incidência e mortalidade, melhorou a qualidade dos dois indicadores, os quais passaram a ter uma taxa suavizada mais estável, segura e livre da influência da densidade populacional, o que foi verificado na diminuição da variabilidade das taxas entre os bairros. $\bigcirc$ método melhora sobremaneira a qualidade dos indicadores, porém essa é a primeira abordagem com aplicação do método bayesiano na Região Norte do Brasil.

A incidência elevada da TB em Belém é preocupante, uma vez que presume transmissão continuada do $M$. tuberculosis associada aos diversos fatores de risco, os quais indicam baixa qualidade de vida e contribuem para gerar a doença no indivíduo infectado. A característica urbana da cidade de Belém apresenta extremos de baixa e alta qualidade de vida em um mesmo bairro, o que promove a alta taxa de incidência observada e a inexistência de grandes diferenças entre os diferentes bairros da cidade. A taxa de mortalidade evidencia uma boa avaliação dos serviços de tratamento e acompanhamento dos doentes, entretanto, é necessária a continuidade de campanhas de prevenção da doença e de acompanhamento para aumentar ainda mais a adesão ao tratamento e a contínua diminuição da taxa de mortalidade entre a população mais carente e com maior dificuldade de acesso aos serviços de saúde.

\section{APOIO FINANCEIRO}

estudo foi parcialmente financiado com recursos do Conselho Nacional de Desenvolvimento Científico e Tecnológico.

\section{CONFLITOS DE INTERESSE}

Os autores declaram não haver conflitos de interesse.

\section{REFERÊNCIAS}

1 World Health Organization. Global tuberculosis report, 2013. Geneva: WHO; 2013.

2 Hiijar MA, Gerhardt G, Teixeira GM, Procópio MJ. Retrospecto do controle da tuberculose no Brasil. Rev Saude Publica. 2007 set;41 Suppl 1:50-8.

3 Xavier MIM, Barreto ML. Tuberculose na cidade de Salvador, Bahia, Brasil: o perfil na década de 1990. Cad Saude Publica. 2007 set;23(2):445-53.

4 Ministério da Saúde (BR). Secretaria de Vigilância em Saúde. Situação da tuberculose no Brasil. Brasília: Ministério da Saúde; 2012.

5 Guimarães RM, Lobo AP, Siqueira EA, Borges TFF, Melo SCC. Tuberculose, HIV e pobreza: tendência temporal no Brasil, Américas e mundo. J Bras Pneumol. 2012 jul-ago;38(4):511-7.

6 Bierrenbach AL, Gomes ABF, Noronha EF, Souza MFM. Incidência de tuberculose e taxa de cura, Brasil, 2000 a 2004. Rev Saude Publica. 2007 set;4 1 Suppl 1:24-33.
7 Roza DL, Caccia-Bava MCGG, Martinez EZ. Spatiotemporal patterns of tuberculosis incidence in Ribeirão Preto, State of São Paulo, southeast Brazil, and their relationship with social vulnerability: a Bayesian analysis. Rev Soc Bras Med Trop. 2012 Sep-Oct;45(5):607-15.

8 Souza WV, Albuquerque MFM, Barcellos CC, Ximenes RAA, Carvalho MS. Tuberculose no Brasil: construção de um sistema de vigilância de base territorial. Rev Saude Publica. 2005 jan;39(1): 82-9.

9 Brunello MEF, Chiaravalloti Neto F, Arcêncio RA, Andrade RLP, Magnabosco GT, Villa TCS. Áreas de vulnerabilidade para co-infecção HIV-aids/ TB em Ribeirão Preto, SP. Rev Saude Publica. 2011 jun;45(3):556-63.

10 Hino P, Villa TCS, Cunha TN, Santos CB. Padrões espaciais da tuberculose e sua associação à condição de vida no município de Ribeirão Preto. Cienc Saude Coletiva. 2011 dez;16(12):4795-802. 
11 Vendramini SHF, Gazetta CE, Chiaravalloti Netto F, Cury MR, Meirelles EB, Kuyumiian FG, et al. Tuberculose em município de porte médio do sudeste do Brasil: indicadores de morbidade e mortalidade, de 1985 a 2003. J Bras Pneumol. 2005 mai-jun;31 (3):237-43.

12 San Pedro A, Oliveira RM. Tuberculose e indicadores socioeconômicos: revisão sistemática da literatura. Rev Panam Salud Publica. 2013 abr;33(4):294-301.

13 Instituto Brasileiro de Geografia e Estatística. Primeiros resultados do Censo 2010 [Internet]. Rio de Janeiro: IBGE; 2010 [citado 2011 ago 16]. Disponível em: http://www.ibge.gov.br/home/estatistica/populacao/ censo2010/primeiros_resultados/populacao_por municipio_zip.shtm.

14 Assunção RM, Barreto SM, Guerra HL, Sakurai E. Mapas de taxas epidemiológicas: uma abordagem Bayesiana. Cad Saude Publica. 1998 out-dez; 14(4):713-23.

15 Anselin L. The Moran scatterplot as an ESDA tool to assess local instability in spatial association. In: Fisher M, Scholten HJ, Unwin, D, editors. Spatial analytical perspectives on GIS. London: Taylor \& Francis; 1996. p. $111-26$.

16 Instituto Brasileiro de Geografia e Estatística. Censo Demográfico 2010 - aglomerados subnormais. Rio de Janeiro: IBGE; 2010.

17 Ayres M, Ayres Jr. M, Ayres DL, Santos AAS. BioEstat 5.0: aplicações estatísticas nas áreas das ciências biológicas e médicas. Belém; Sociedade Civil Mamirauá; 2007. 364 p.

18 Ministério da Saúde (BR). Departamento de Informática do SUS. Informações de Saúde: indicadores de morbidade. Brasília: Ministério da Saúde; 2013.

19 Vieira RCA, Prado TN, Siqueira MG, Dietze R, Maciel ELN. Distribuição espacial dos casos novos de tuberculose em Vitória, Estado do Espírito Santo, no período entre 2000 e 2005. Rev Soc Bras Med Trop. 2008 jan-fev; $41(1): 82-6$.

20 Souza WV, Carvalho MS, Albuquerque MFPM, Barcellos CC, Ximenes RAA. Tuberculosis in intraurban settings: a Bayesian approach. Trop Med Int Health. 2007 Mar; 12(3):323-30.
21 Maciel ELN, Pan W, Dietze R, Peres RL, Vinhas SA, Ribeiro FK, et al. Spatial patterns of pulmonary tuberculosis incidence and their relationship to socioeconomic status in Vitoria, Brazil. Int J Tuberc Lung Dis. 2010 Nov; $14(11): 1395-402$.

22 Vendramini SHF, Santos NSGM, Santos MLSG, Chiaravalloti-Neto F, Ponce MAS, Gazetta CE, et al. Análise espacial da co-infecção tuberculose/HIV: relação com níveis socioeconômicos em município do sudeste do Brasil. Rev Soc Bras Med Trop. 2010 set-out;43(5):536-41.

23 Sales CMM, Figueiredo TAM, Zandonade E, Maciel ELN. Análise espacial da tuberculose infantil no estado do Espírito Santo, 2000 a 2007. Rev Soc Bras Med Trop. 2010 jul-ago;43(4):435-9.

24 Hino P, Santos CB, Villa TCS. Evolução espaçotemporal dos casos de tuberculose em Ribeirão Preto (SP), nos anos de 1998 a 2002. J Bras Pneumol. 2005 nov-dez;31 (6):523-7.

25 Queiroga RPF, Sá LD, Nogueira JA, Lima ERV, Silva ACO, Pinheiro PGOD, et al. Distribuição espacial da tuberculose e a relação com condições de vida na área urbana do município de Campina Grande 2004 a 2007. Rev Bras Epidemiol. 2012 mar;15(1): 222-32.

26 Golub JE, Bur S, Cronin WA, Gange S, Baruch N, Comstock GW, et al. Delayed tuberculosis diagnosis and tuberculosis transmission. Int J Tuberc Lung Dis. 2006 Jan; 10(1):24-30.

27 Machado ACFT, Steffen RE, Oxlade O, Menzies D, Kritski A, Trajman A. Fatores associados ao atraso no diagnóstico da tuberculose pulmonar no estado do Rio de Janeiro. J Bras Pneumol. 2011 jul-ago;37(4):512-20.

28 Mota FF, Vieira-da-Silva LM, Paim JS, Costa MCN. Distribuição espacial da mortalidade por tuberculose em Salvador, Bahia, Brasil. Cad Saude Publica. 2003 jul-ago; 19(4):915-22. 